\title{
X-ray and UV Spectra of Glycine within Coupled Cluster Linear Response Theory; Supplemental Material
}

\author{
Rolf H. Myhre, ${ }^{\dagger} \S$ Sonia Coriani, ${ }^{*,+, \|}$ and Henrik Koch*,†, \\ $\dagger$ Department of Chemistry, Norwegian University of Science and Technology, NTNU, 7491 \\ Trondheim, Norway \\ $\ddagger D T U$ Chemistry, Technical University of Denmark, DK-2800 Kongens Lyngby, Denmark \\ 9Scuola Normale Superiore, Piazza dei Cavaleri 7, 56126 Pisa, Italy \\ $\S$ Hylleraas Centre for Quantum Molecular Sciences, Department of Chemistry, University \\ of Oslo, 0315 Oslo, Norway \\ ||Aarhus Institute of Avanced Studies, University of Aarhus, DK-8000 Århus C, Denmark \\ E-mail: soco@kemi.dtu.dk; henrik.koch@sns.it
}




\section{Conformer geometries in Ångström}

Table S1: Conformer I

\begin{tabular}{rrrr}
\hline & \multicolumn{1}{c}{$\mathrm{X}$} & \multicolumn{1}{c}{$\mathrm{Y}$} & \multicolumn{1}{c}{$\mathrm{Z}$} \\
\hline $\mathrm{H}$ & 2.38124117 & -0.10062141 & 0.00144789 \\
$\mathrm{O}$ & 1.61785794 & -0.70346926 & 0.00122800 \\
$\mathrm{O}$ & 0.55188249 & 1.30549510 & -0.00060016 \\
$\mathrm{C}$ & 0.50292531 & 0.08780740 & -0.00024565 \\
$\mathrm{C}$ & -0.77208965 & -0.75453924 & -0.00222208 \\
$\mathrm{H}$ & -0.73395636 & -1.41946269 & 0.87889853 \\
$\mathrm{H}$ & -0.73499644 & -1.41250151 & -0.88866228 \\
$\mathrm{~N}$ & -2.02089069 & 0.00429124 & 0.00132666 \\
$\mathrm{H}$ & -2.03227510 & 0.62419079 & 0.81514250 \\
$\mathrm{H}$ & -2.03151172 & 0.63284551 & -0.80584108 \\
\hline
\end{tabular}

Table S2: Conformer II

\begin{tabular}{rrrr}
\hline & $\mathrm{X}$ & \multicolumn{1}{c}{$\mathrm{Y}$} & \multicolumn{1}{c}{$\mathrm{Z}$} \\
\hline $\mathrm{C}$ & 0.61087527 & 0.04640133 & -0.00014205 \\
$\mathrm{O}$ & 1.69863348 & 0.59001876 & 0.00176200 \\
$\mathrm{O}$ & 0.45331233 & -1.29766282 & -0.00115861 \\
$\mathrm{H}$ & -0.52274718 & -1.42961977 & -0.00253879 \\
$\mathrm{C}$ & -0.71089707 & 0.83758501 & -0.00268757 \\
$\mathrm{H}$ & -0.69584274 & 1.48633388 & -0.89422607 \\
$\mathrm{H}$ & -0.69387052 & 1.49702743 & 0.88094500 \\
$\mathrm{~N}$ & -1.87967736 & -0.07171859 & 0.00188157 \\
$\mathrm{H}$ & -2.47239940 & 0.07766456 & -0.81327260 \\
$\mathrm{H}$ & -2.46024391 & 0.07042802 & 0.82706486 \\
\hline
\end{tabular}


Table S3: Conformer III

\begin{tabular}{rrrr}
\hline & \multicolumn{1}{c}{$\mathrm{X}$} & \multicolumn{1}{c}{$\mathrm{Y}$} & \multicolumn{1}{c}{$\mathrm{Z}$} \\
\hline $\mathrm{C}$ & 0.58519094 & -0.08210731 & -0.00062669 \\
$\mathrm{O}$ & 1.65478101 & -0.66639287 & 0.00588468 \\
$\mathrm{O}$ & 0.48766365 & 1.28274843 & -0.00330776 \\
$\mathrm{H}$ & 1.40458712 & 1.60903115 & 0.00030588 \\
$\mathrm{C}$ & -0.77555087 & -0.78429841 & -0.00984230 \\
$\mathrm{H}$ & -0.77732115 & -1.46266678 & 0.86090709 \\
$\mathrm{H}$ & -0.78064545 & -1.43119364 & -0.90447455 \\
$\mathrm{~N}$ & -1.98734754 & 0.03659623 & 0.00641079 \\
$\mathrm{H}$ & -1.98436879 & 0.67172983 & -0.79430915 \\
$\mathrm{H}$ & -1.98491733 & 0.63875284 & 0.83225185 \\
\hline
\end{tabular}

Table S4: Conformer IV

\begin{tabular}{rrrr}
\hline & \multicolumn{1}{c}{$\mathrm{X}$} & \multicolumn{1}{c}{$\mathrm{Y}$} & \multicolumn{1}{c}{$\mathrm{Z}$} \\
\hline $\mathrm{C}$ & 0.49241984 & -0.08181555 & -0.02384371 \\
$\mathrm{O}$ & 0.53345764 & -1.29937684 & -0.04861085 \\
$\mathrm{O}$ & 1.60453398 & 0.70166373 & 0.08963844 \\
$\mathrm{H}$ & 2.36241304 & 0.09254265 & 0.12134732 \\
$\mathrm{C}$ & -0.76773451 & 0.75428431 & -0.16581131 \\
$\mathrm{H}$ & -0.68641582 & 1.62712841 & 0.50367344 \\
$\mathrm{H}$ & -0.75673678 & 1.13827344 & -1.20877989 \\
$\mathrm{~N}$ & -1.93950915 & -0.05487834 & 0.18880611 \\
$\mathrm{H}$ & -2.78427976 & 0.36581410 & -0.20059310 \\
$\mathrm{H}$ & -1.84011512 & -0.98208926 & -0.23193365 \\
\hline
\end{tabular}

Table S5: Conformer V

\begin{tabular}{rrrr}
\hline & \multicolumn{1}{c}{$\mathrm{X}$} & \multicolumn{1}{c}{$\mathrm{Y}$} & \multicolumn{1}{c}{$\mathrm{Z}$} \\
\hline $\mathrm{C}$ & 0.56563596 & -0.09532450 & -0.03536142 \\
$\mathrm{O}$ & 1.58060770 & -0.72952708 & 0.18979502 \\
$\mathrm{O}$ & 0.54293366 & 1.27114802 & -0.09994068 \\
$\mathrm{H}$ & 1.45191418 & 1.56411840 & 0.08424110 \\
$\mathrm{C}$ & -0.79900077 & -0.69333828 & -0.34058579 \\
$\mathrm{H}$ & -0.79001712 & -1.72216619 & 0.05013899 \\
$\mathrm{H}$ & -0.87261332 & -0.74717005 & -1.44846940 \\
$\mathrm{~N}$ & -1.87468404 & 0.07330656 & 0.31282071 \\
$\mathrm{H}$ & -2.77612290 & -0.34509310 & 0.07499391 \\
$\mathrm{H}$ & -1.88915188 & 1.02631933 & -0.05705960 \\
\hline
\end{tabular}


Table S6: Relative ground state energies with and without zero point energies in $\mathrm{kJ} / \mathrm{mol}, \mathrm{CCSD}(\mathrm{T})$, aug-cc-pVDZ, $\mathrm{kJ} / \mathrm{mol}$. Boltzmann weights at $298 \mathrm{~K}$

\begin{tabular}{crcrc}
\hline & \multicolumn{1}{c}{$\Delta \mathrm{E}_{0}$} & $\mathrm{~V}_{0}$ & total & $\mathrm{w}$ \\
\hline $\mathrm{I}$ & 0.0000 & 208.228 & 0.0000 & 0.732 \\
$\mathrm{II}$ & 3.2901 & 209.190 & 4.2520 & 0.132 \\
$\mathrm{III}$ & 6.8944 & 208.329 & 6.9957 & 0.044 \\
$\mathrm{IV}$ & 5.4620 & 208.200 & 5.4338 & 0.082 \\
$\mathrm{~V}$ & 10.2096 & 208.512 & 10.4934 & 0.011 \\
\hline
\end{tabular}




\section{Conformer I}

Table S7: Conformer I - valence excitations and ionizations (eV).

\begin{tabular}{|c|c|c|c|c|c|c|c|c|}
\hline & \multicolumn{4}{|c|}{ CCSD } & \multicolumn{2}{|c|}{ CC3 } & \multirow{3}{*}{$\begin{array}{c}\text { exp. } \\
( \pm 0.1)^{1} \\
\Delta \mathrm{E}\end{array}$} & \multirow{3}{*}{ Asgn } \\
\hline & \multicolumn{2}{|c|}{$\mathrm{aCDZ}$} & \multicolumn{2}{|c|}{ aCTZ } & \multirow{2}{*}{$\frac{\mathrm{aCDZ}}{\Delta \mathrm{E}}$} & \multirow{2}{*}{$\frac{\mathrm{aCTZ}}{\Delta \mathrm{E}}$} & & \\
\hline & $\Delta \mathrm{E}$ & $f \times 100$ & $\Delta \mathrm{E}$ & $f \times 100$ & & & & \\
\hline $\mathrm{S}_{1}$ & $5.90\left(\mathrm{~A}^{\prime \prime}\right)$ & 0.01 & $5.89\left(\mathrm{~A}^{\prime \prime}\right)$ & 0.02 & $5.88\left(\mathrm{~A}^{\prime \prime}\right)$ & $5.83\left(\mathrm{~A}^{\prime \prime}\right)$ & - & \\
\hline $\mathrm{S}_{2}$ & $6.45\left(\mathrm{~A}^{\prime}\right)$ & 1.62 & $6.62\left(\mathrm{~A}^{\prime}\right)$ & 1.57 & $6.39\left(\mathrm{~A}^{\prime}\right)$ & $6.51\left(\mathrm{~A}^{\prime}\right)$ & - & \\
\hline $\mathrm{S}_{3}$ & $7.27\left(\mathrm{~A}^{\prime}\right)$ & 7.72 & $7.47\left(\mathrm{~A}^{\prime}\right)$ & 7.44 & $7.16\left(\mathrm{~A}^{\prime}\right)$ & $7.15\left(\mathrm{~A}^{\prime \prime}\right)$ & - & \\
\hline $\mathrm{S}_{4}$ & $7.37\left(\mathrm{~A}^{\prime \prime}\right)$ & 0.06 & $7.47\left(\mathrm{~A}^{\prime \prime}\right)$ & 0.03 & $7.17\left(\mathrm{~A}^{\prime \prime}\right)$ & $7.27\left(\mathrm{~A}^{\prime}\right)$ & - & \\
\hline $\mathrm{S}_{5}$ & $7.57\left(\mathrm{~A}^{\prime}\right)$ & 1.01 & $7.74\left(\mathrm{~A}^{\prime}\right)$ & 1.06 & $7.49\left(\mathrm{~A}^{\prime}\right)$ & - & - & \\
\hline $\mathrm{I}_{1}$ & $10.01\left(\mathrm{~A}^{\prime}\right)$ & - & $10.25\left(\mathrm{~A}^{\prime}\right)$ & - & $9.97\left(\mathrm{~A}^{\prime}\right)$ & $10.13\left(\mathrm{~A}^{\prime}\right)$ & 10.0 & $\mathrm{n}_{N}$ \\
\hline $\mathrm{I}_{2}$ & $10.90\left(\mathrm{~A}^{\prime}\right)$ & - & $11.16\left(\mathrm{~A}^{\prime}\right)$ & - & $10.88\left(\mathrm{~A}^{\prime}\right)$ & - & 11.2 & $\mathrm{n}_{O}$ \\
\hline $\mathrm{I}_{3}$ & $12.06\left(\mathrm{~A}^{\prime \prime}\right)$ & - & $12.31\left(\mathrm{~A}^{\prime \prime}\right)$ & - & $12.01\left(\mathrm{~A}^{\prime \prime}\right)$ & - & 12.2 & $\pi_{O O}$ \\
\hline $\mathrm{I}_{4}$ & $13.53\left(\mathrm{~A}^{\prime \prime}\right)$ & - & $13.71\left(\mathrm{~A}^{\prime \prime}\right)$ & - & $13.41\left(\mathrm{~A}^{\prime \prime}\right)$ & - & 13.7 & \\
\hline $\mathrm{I}_{5}$ & $14.37\left(\mathrm{~A}^{\prime}\right)$ & - & $14.57\left(\mathrm{~A}^{\prime}\right)$ & - & $14.27\left(\mathrm{~A}^{\prime}\right)$ & - & 14.4 & \\
\hline
\end{tabular}


Table S8: Conformer I. CCSD core excitation energies (in eV) and corresponding intensities $(\times 100)$.

\begin{tabular}{|c|c|c|c|c|c|c|c|c|}
\hline & \multicolumn{2}{|c|}{ aCDZ } & \multicolumn{2}{|c|}{$\mathrm{a}(\mathrm{CT}) \mathrm{Z}$} & \multicolumn{2}{|c|}{ aCTZ } & \multicolumn{2}{|c|}{$\mathrm{a}(\mathrm{CQ}) \mathrm{Z}$} \\
\hline & $\Delta \mathrm{E}$ & $f \times 10^{2}$ & $\Delta \mathrm{E}$ & $f \times 10^{2}$ & $\Delta \mathrm{E}$ & $f \times 10^{2}$ & $\Delta \mathrm{E}$ & $f \times 10^{2}$ \\
\hline \multirow{5}{*}{$\mathrm{C} 1$} & $290.79\left(\mathrm{~A}^{\prime}\right)$ & 0.96 & $289.85\left(\mathrm{~A}^{\prime}\right)$ & 1.04 & $289.87\left(\mathrm{~A}^{\prime}\right)$ & 1.04 & $289.79\left(\mathrm{~A}^{\prime}\right)$ & 1.07 \\
\hline & $291.03\left(\mathrm{~A}^{\prime \prime}\right)$ & 0.58 & $290.07\left(\mathrm{~A}^{\prime \prime}\right)$ & 0.62 & $290.00\left(\mathrm{~A}^{\prime \prime}\right)$ & 0.51 & $290.00\left(\mathrm{~A}^{\prime \prime}\right)$ & 0.65 \\
\hline & $291.96\left(\mathrm{~A}^{\prime}\right)$ & 0.34 & $291.01\left(\mathrm{~A}^{\prime}\right)$ & 0.38 & $291.01\left(\mathrm{~A}^{\prime \prime}\right)$ & 1.24 & $290.95\left(\mathrm{~A}^{\prime}\right)$ & 0.40 \\
\hline & $291.98\left(\mathrm{~A}^{\prime \prime}\right)$ & 1.19 & $291.04\left(\mathrm{~A}^{\prime \prime}\right)$ & 1.24 & $291.02\left(\mathrm{~A}^{\prime}\right)$ & 0.48 & $290.97\left(\mathrm{~A}^{\prime \prime}\right)$ & 1.28 \\
\hline & $292.41\left(\mathrm{~A}^{\prime}\right)$ & 0.65 & $291.45\left(\mathrm{~A}^{\prime}\right)$ & 0.64 & $291.46\left(\mathrm{~A}^{\prime}\right)$ & 0.64 & $291.39\left(\mathrm{~A}^{\prime}\right)$ & 0.64 \\
\hline \multirow{5}{*}{$\mathrm{C} 2$} & $290.53\left(\mathrm{~A}^{\prime \prime}\right)$ & 7.20 & $289.48\left(\mathrm{~A}^{\prime \prime}\right)$ & 7.55 & $289.43\left(\mathrm{~A}^{\prime \prime}\right)$ & 7.62 & $289.35\left(\mathrm{~A}^{\prime \prime}\right)$ & 7.64 \\
\hline & $294.35\left(\mathrm{~A}^{\prime}\right)$ & 0.10 & $293.40\left(\mathrm{~A}^{\prime}\right)$ & 0.10 & $293.44\left(\mathrm{~A}^{\prime}\right)$ & 0.11 & $293.35\left(\mathrm{~A}^{\prime}\right)$ & 0.11 \\
\hline & $295.23\left(\mathrm{~A}^{\prime}\right)$ & 0.14 & $294.28\left(\mathrm{~A}^{\prime}\right)$ & 0.15 & $294.30\left(\mathrm{~A}^{\prime}\right)$ & 0.15 & $294.22\left(\mathrm{~A}^{\prime}\right)$ & 0.16 \\
\hline & $295.63\left(\mathrm{~A}^{\prime \prime}\right)$ & 0.03 & $294.66\left(\mathrm{~A}^{\prime \prime}\right)$ & 0.02 & $294.67\left(\mathrm{~A}^{\prime \prime}\right)$ & 0.02 & $294.59\left(\mathrm{~A}^{\prime \prime}\right)$ & 0.02 \\
\hline & $295.84\left(\mathrm{~A}^{\prime}\right)$ & 0.02 & $294.89\left(\mathrm{~A}^{\prime}\right)$ & 0.02 & $294.89\left(\mathrm{~A}^{\prime}\right)$ & 0.02 & $294.82\left(\mathrm{~A}^{\prime}\right)$ & 0.03 \\
\hline \multirow{5}{*}{ N3 } & $404.30\left(\mathrm{~A}^{\prime}\right)$ & 0.60 & $402.90\left(\mathrm{~A}^{\prime}\right)$ & 0.61 & $402.90\left(\mathrm{~A}^{\prime}\right)$ & 0.61 & $402.82\left(\mathrm{~A}^{\prime}\right)$ & 0.63 \\
\hline & $405.41\left(\mathrm{~A}^{\prime \prime}\right)$ & 1.89 & $403.99\left(\mathrm{~A}^{\prime \prime}\right)$ & 1.95 & $403.99\left(\mathrm{~A}^{\prime \prime}\right)$ & 1.95 & $403.90\left(\mathrm{~A}^{\prime \prime}\right)$ & 2.00 \\
\hline & $405.94\left(\mathrm{~A}^{\prime}\right)$ & 0.10 & $404.52\left(\mathrm{~A}^{\prime}\right)$ & 0.11 & $404.51\left(\mathrm{~A}^{\prime}\right)$ & 0.11 & $404.45\left(\mathrm{~A}^{\prime}\right)$ & 0.12 \\
\hline & $406.49\left(\mathrm{~A}^{\prime \prime}\right)$ & 0.32 & $405.08\left(\mathrm{~A}^{\prime \prime}\right)$ & 0.33 & $405.00\left(\mathrm{~A}^{\prime \prime}\right)$ & 0.31 & $405.00\left(\mathrm{~A}^{\prime \prime}\right)$ & 0.33 \\
\hline & $406.57\left(\mathrm{~A}^{\prime}\right)$ & 0.59 & $405.13\left(\mathrm{~A}^{\prime}\right)$ & 0.62 & $405.05\left(\mathrm{~A}^{\prime}\right)$ & 0.58 & $405.01\left(\mathrm{~A}^{\prime}\right)$ & 0.64 \\
\hline \multirow{5}{*}{$\mathrm{O} 4$} & $535.87\left(\mathrm{~A}^{\prime \prime}\right)$ & 3.75 & $533.96\left(\mathrm{~A}^{\prime \prime}\right)$ & 3.78 & $533.90\left(\mathrm{~A}^{\prime \prime}\right)$ & 3.82 & $533.83\left(\mathrm{~A}^{\prime \prime}\right)$ & 3.79 \\
\hline & $539.09\left(\mathrm{~A}^{\prime}\right)$ & 0.08 & $537.14\left(\mathrm{~A}^{\prime}\right)$ & 0.08 & $537.17\left(\mathrm{~A}^{\prime}\right)$ & 0.08 & $537.04\left(\mathrm{~A}^{\prime}\right)$ & 0.08 \\
\hline & $540.01\left(\mathrm{~A}^{\prime}\right)$ & 0.44 & $538.06\left(\mathrm{~A}^{\prime}\right)$ & 0.43 & $538.09\left(\mathrm{~A}^{\prime}\right)$ & 0.43 & $537.96\left(\mathrm{~A}^{\prime}\right)$ & 0.43 \\
\hline & $540.39\left(\mathrm{~A}^{\prime \prime}\right)$ & 0.13 & $538.42\left(\mathrm{~A}^{\prime \prime}\right)$ & 0.14 & $538.40\left(\mathrm{~A}^{\prime \prime}\right)$ & 0.14 & $538.33\left(\mathrm{~A}^{\prime \prime}\right)$ & 0.15 \\
\hline & $540.70\left(\mathrm{~A}^{\prime}\right)$ & 0.19 & $538.74\left(\mathrm{~A}^{\prime}\right)$ & 0.20 & $538.71\left(\mathrm{~A}^{\prime}\right)$ & 0.19 & $538.62\left(\mathrm{~A}^{\prime}\right)$ & 0.20 \\
\hline \multirow{5}{*}{ O5 } & $539.19\left(\mathrm{~A}^{\prime}\right)$ & 1.56 & $537.34\left(\mathrm{~A}^{\prime}\right)$ & 1.55 & $537.37\left(\mathrm{~A}^{\prime}\right)$ & 1.56 & $537.23\left(\mathrm{~A}^{\prime}\right)$ & 1.58 \\
\hline & $539.75\left(\mathrm{~A}^{\prime \prime}\right)$ & 1.46 & $537.89\left(\mathrm{~A}^{\prime \prime}\right)$ & 1.45 & $537.79\left(\mathrm{~A}^{\prime \prime}\right)$ & 1.46 & $537.78\left(\mathrm{~A}^{\prime \prime}\right)$ & 1.46 \\
\hline & $541.37\left(\mathrm{~A}^{\prime}\right)$ & 0.76 & $539.49\left(\mathrm{~A}^{\prime}\right)$ & 0.75 & $539.47\left(\mathrm{~A}^{\prime}\right)$ & 0.75 & $539.38\left(\mathrm{~A}^{\prime}\right)$ & 0.76 \\
\hline & $541.74\left(\mathrm{~A}^{\prime \prime}\right)$ & 0.37 & $539.85\left(\mathrm{~A}^{\prime \prime}\right)$ & 0.40 & $539.80\left(\mathrm{~A}^{\prime \prime}\right)$ & 0.38 & $539.75\left(\mathrm{~A}^{\prime \prime}\right)$ & 0.41 \\
\hline & $542.05\left(\mathrm{~A}^{\prime}\right)$ & 0.36 & $540.15\left(\mathrm{~A}^{\prime}\right)$ & 0.36 & $540.11\left(\mathrm{~A}^{\prime}\right)$ & 0.32 & $540.04\left(\mathrm{~A}^{\prime}\right)$ & 0.34 \\
\hline
\end{tabular}


Table S9: Conformer I. CC3 core excitation energies and experimental results from Plekan et al. ${ }^{2}(\mathrm{eV})$. The point group symmetry could not be determined for the degenerate states of $\mathrm{C} 1$ with aCDZ

\begin{tabular}{|c|c|c|c|c|c|}
\hline & $\mathrm{aCDZ}$ & $\mathrm{a}(\mathrm{CT}) \mathrm{Z}$ & $\mathrm{aCTZ}$ & $\mathrm{a}(\mathrm{CQ}) \mathrm{Z}$ & exp. \\
\hline $\mathrm{C} 1$ & $\begin{array}{l}289.77\left(\mathrm{~A}^{*}\right) \\
289.77\left(\mathrm{~A}^{*}\right) \\
290.74\left(\mathrm{~A}^{\prime}\right) \\
290.83\left(\mathrm{~A}^{\prime \prime}\right) \\
291.06\left(\mathrm{~A}^{\prime}\right)\end{array}$ & $\begin{array}{l}288.71\left(\mathrm{~A}^{\prime \prime}\right) \\
288.73\left(\mathrm{~A}^{\prime}\right) \\
289.70\left(\mathrm{~A}^{\prime}\right) \\
289.79\left(\mathrm{~A}^{\prime \prime}\right) \\
290.02\left(\mathrm{~A}^{\prime}\right)\end{array}$ & $288.59\left(\mathrm{~A}^{\prime \prime}\right)$ & $288.57\left(\mathrm{~A}^{\prime \prime}\right)$ & 289.4 \\
\hline $\mathrm{C} 2$ & $\begin{array}{l}290.15\left(\mathrm{~A}^{\prime \prime}\right) \\
293.09\left(\mathrm{~A}^{\prime}\right) \\
293.86\left(\mathrm{~A}^{\prime}\right) \\
294.35\left(\mathrm{~A}^{\prime \prime}\right) \\
294.47\left(\mathrm{~A}^{\prime}\right)\end{array}$ & $\begin{array}{l}288.97\left(\mathrm{~A}^{\prime \prime}\right) \\
292.03\left(\mathrm{~A}^{\prime}\right) \\
292.81\left(\mathrm{~A}^{\prime}\right) \\
293.27\left(\mathrm{~A}^{\prime \prime}\right) \\
293.41\left(\mathrm{~A}^{\prime}\right)\end{array}$ & $288.94\left(\mathrm{~A}^{\prime \prime}\right)$ & $288.78\left(\mathrm{~A}^{\prime \prime}\right)$ & $\begin{array}{l}291.3 \\
288.4\end{array}$ \\
\hline N3 & $\begin{array}{l}402.76\left(\mathrm{~A}^{\prime}\right) \\
403.76\left(\mathrm{~A}^{\prime \prime}\right) \\
403.94\left(\mathrm{~A}^{\prime}\right) \\
403.94\left(\mathrm{~A}^{\prime \prime}\right) \\
404.55\left(\mathrm{~A}^{\prime}\right)\end{array}$ & $\begin{array}{l}401.34\left(\mathrm{~A}^{\prime}\right) \\
402.36\left(\mathrm{~A}^{\prime \prime}\right) \\
402.54\left(\mathrm{~A}^{\prime}\right) \\
402.55\left(\mathrm{~A}^{\prime \prime}\right) \\
403.15\left(\mathrm{~A}^{\prime}\right)\end{array}$ & $401.34\left(\mathrm{~A}^{\prime}\right)$ & $401.20\left(\mathrm{~A}^{\prime}\right)$ & $\begin{array}{l}401.2 \\
402.4 \\
403.8 \\
404.8\end{array}$ \\
\hline $\mathrm{O} 4$ & $\begin{array}{l}533.94\left(\mathrm{~A}^{\prime \prime}\right) \\
535.63\left(\mathrm{~A}^{\prime}\right) \\
536.43\left(\mathrm{~A}^{\prime}\right) \\
536.83\left(\mathrm{~A}^{\prime}\right) \\
537.01\left(\mathrm{~A}^{\prime}\right)\end{array}$ & $\begin{array}{l}532.10\left(\mathrm{~A}^{\prime \prime}\right) \\
533.93\left(\mathrm{~A}^{\prime}\right) \\
534.73\left(\mathrm{~A}^{\prime}\right) \\
535.13\left(\mathrm{~A}^{\prime \prime}\right) \\
535.31\left(\mathrm{~A}^{\prime}\right)\end{array}$ & $532.01\left(\mathrm{~A}^{\prime \prime}\right)$ & $531.90\left(\mathrm{~A}^{\prime \prime}\right)$ & 532.2 \\
\hline $\mathrm{O} 5$ & $\begin{array}{l}536.86\left(\mathrm{~A}^{\prime \prime}\right) \\
536.92\left(\mathrm{~A}^{\prime}\right) \\
538.30\left(\mathrm{~A}^{\prime}\right) \\
538.74\left(\mathrm{~A}^{\prime \prime}\right) \\
538.90\left(\mathrm{~A}^{\prime}\right)\end{array}$ & $\begin{array}{l}535.16\left(\mathrm{~A}^{\prime \prime}\right) \\
535.22\left(\mathrm{~A}^{\prime}\right) \\
536.65\left(\mathrm{~A}^{\prime}\right) \\
537.08\left(\mathrm{~A}^{\prime \prime}\right) \\
537.24\left(\mathrm{~A}^{\prime}\right)\end{array}$ & $535.01\left(\mathrm{~A}^{\prime \prime}\right)$ & $534.99\left(\mathrm{~A}^{\prime \prime}\right)$ & $\begin{array}{l}539.2 \\
535.4\end{array}$ \\
\hline
\end{tabular}

Table S10: Conformer I CCSD core ionization energies (eV)

\begin{tabular}{ccccc}
\hline & $\mathrm{aCDZ}$ & $\mathrm{aC}(\mathrm{T}) \mathrm{Z}$ & $\mathrm{aCTZ}$ & $\mathrm{aC}(\mathrm{Q}) \mathrm{Z}$ \\
\hline $\mathrm{C} 1$ & 294.68 & 293.75 & 293.79 & 293.70 \\
$\mathrm{C} 2$ & 297.92 & 296.98 & 297.04 & 296.93 \\
$\mathrm{~N} 3$ & 408.56 & 407.14 & 407.17 & 407.08 \\
$\mathrm{O} 4$ & 542.71 & 540.75 & 540.81 & 540.66 \\
$\mathrm{O} 5$ & 544.22 & 542.33 & 542.38 & 542.24 \\
\hline
\end{tabular}


Table S11: Conformer I CC3 core ionization energies and experimental results from Plekan et al. ${ }^{1}(\mathrm{eV})$

\begin{tabular}{cccccc}
\hline & $\mathrm{aCDZ}$ & $\mathrm{aC}(\mathrm{T}) \mathrm{Z}$ & $\mathrm{aCTZ}$ & $\mathrm{aC}(\mathrm{Q}) \mathrm{Z}$ & exp. $( \pm 0.1)$ \\
\hline $\mathrm{C} 1$ & 293.37 & 292.35 & 292.40 & 292.23 & 292.3 \\
$\mathrm{C} 2$ & 296.57 & 295.52 & 295.58 & 295.39 & 295.2 \\
$\mathrm{~N} 3$ & 406.48 & 405.09 & 405.11 & 404.95 & 405.4 \\
$\mathrm{O} 4$ & 538.91 & 537.24 & 537.29 & 537.09 & 538.4 \\
$\mathrm{O} 5$ & 540.83 & 539.21 & 539.24 & 539.05 & 540.2 \\
\hline
\end{tabular}




\section{Conformers, ionizations and valence excitations}

Table S12: Glycine CCSD valence excitations (eV), aCDZ

\begin{tabular}{ccccccccccc}
\hline & \multicolumn{3}{c}{$\mathrm{I}$} & \multicolumn{2}{c}{$\mathrm{II}$} & \multicolumn{2}{c}{$\mathrm{III}$} & \multicolumn{3}{c}{$\mathrm{V}$} \\
& $\Delta \mathrm{E}$ & $f \times 100$ & $\Delta \mathrm{E}$ & $f \times 100$ & $\Delta \mathrm{E}$ & $f \times 100$ & $\Delta \mathrm{E}$ & $f \times 100$ & $\Delta \mathrm{E}$ & $f \times 100$ \\
\hline $\mathrm{S}_{1}$ & 5.90 & $0.01\left(\mathrm{~A}^{\prime \prime}\right)$ & 5.74 & $0.02\left(\mathrm{~A}^{\prime \prime}\right)$ & 5.67 & $0.01\left(\mathrm{~A}^{\prime \prime}\right)$ & 5.94 & 0.03 & 5.74 & 0.05 \\
$\mathrm{~S}_{2}$ & 6.45 & $1.62\left(\mathrm{~A}^{\prime}\right)$ & 6.37 & $1.15\left(\mathrm{~A}^{\prime}\right)$ & 6.15 & $0.84\left(\mathrm{~A}^{\prime}\right)$ & 6.16 & 1.46 & 6.19 & 1.38 \\
$\mathrm{~S}_{3}$ & 7.27 & $7.72\left(\mathrm{~A}^{\prime}\right)$ & 7.24 & $7.18\left(\mathrm{~A}^{\prime}\right)$ & 7.05 & $7.95\left(\mathrm{~A}^{\prime}\right)$ & 7.25 & 3.31 & 7.20 & 4.66 \\
$\mathrm{~S}_{4}$ & 7.37 & $0.06\left(\mathrm{~A}^{\prime \prime}\right)$ & 7.48 & $0.01\left(\mathrm{~A}^{\prime \prime}\right)$ & 7.22 & $0.05\left(\mathrm{~A}^{\prime \prime}\right)$ & 7.33 & 1.16 & 7.37 & 1.39 \\
$\mathrm{~S}_{5}$ & 7.57 & $1.01\left(\mathrm{~A}^{\prime}\right)$ & 7.72 & $1.53\left(\mathrm{~A}^{\prime}\right)$ & 7.35 & $0.95\left(\mathrm{~A}^{\prime}\right)$ & 7.42 & 3.66 & 7.50 & 2.85 \\
$\mathrm{~S}_{6}$ & 7.68 & $0.10\left(\mathrm{~A}^{\prime \prime}\right)$ & 7.92 & $0.35\left(\mathrm{~A}^{\prime}\right)$ & 7.92 & $0.02\left(\mathrm{~A}^{\prime \prime}\right)$ & 7.57 & 1.96 & 7.68 & 2.81 \\
$\mathrm{~S}_{7}$ & 8.04 & $0.68\left(\mathrm{~A}^{\prime}\right)$ & 8.01 & $0.26\left(\mathrm{~A}^{\prime \prime}\right)$ & 8.15 & $0.30\left(\mathrm{~A}^{\prime \prime}\right)$ & 7.88 & 3.94 & 7.92 & 2.65 \\
$\mathrm{~S}_{8}$ & 8.21 & $4.50\left(\mathrm{~A}^{\prime}\right)$ & 8.24 & $11.75\left(\mathrm{~A}^{\prime}\right)$ & 8.23 & $1.83\left(\mathrm{~A}^{\prime}\right)$ & 8.14 & 3.21 & 8.19 & 2.59 \\
$\mathrm{~S}_{9}$ & 8.28 & $0.30\left(\mathrm{~A}^{\prime \prime}\right)$ & 8.31 & $0.00\left(\mathrm{~A}^{\prime \prime}\right)$ & 8.26 & $1.83\left(\mathrm{~A}^{\prime}\right)$ & 8.21 & 0.81 & 8.31 & 0.19 \\
$\mathrm{~S}_{10}$ & 8.56 & $0.01\left(\mathrm{~A}^{\prime \prime}\right)$ & 8.52 & $4.55\left(\mathrm{~A}^{\prime}\right)$ & 8.38 & $8.82\left(\mathrm{~A}^{\prime}\right)$ & 8.42 & 0.17 & 8.44 & 5.80 \\
\hline
\end{tabular}

Table S13: Glycine CC3 valence excitations (eV), aCDZ

\begin{tabular}{cccccc}
\hline & I & II & III & IV & V \\
\hline $\mathrm{S}_{1}$ & $5.88\left(\mathrm{~A}^{\prime \prime}\right)$ & $5.72\left(\mathrm{~A}^{\prime \prime}\right)$ & $5.62\left(\mathrm{~A}^{\prime \prime}\right)$ & 5.92 & 5.71 \\
$\mathrm{~S}_{2}$ & $6.39\left(\mathrm{~A}^{\prime}\right)$ & $6.26\left(\mathrm{~A}^{\prime}\right)$ & $6.07\left(\mathrm{~A}^{\prime}\right)$ & 6.12 & 6.13 \\
$\mathrm{~S}_{3}$ & $7.16\left(\mathrm{~A}^{\prime}\right)$ & $7.16\left(\mathrm{~A}^{\prime}\right)$ & $6.95\left(\mathrm{~A}^{\prime}\right)$ & 7.08 & 7.07 \\
$\mathrm{~S}_{4}$ & $7.17\left(\mathrm{~A}^{\prime \prime}\right)$ & $7.40\left(\mathrm{~A}^{\prime \prime}\right)$ & $7.16\left(\mathrm{~A}^{\prime \prime}\right)$ & 7.17 & 7.31 \\
$\mathrm{~S}_{5}$ & $7.49\left(\mathrm{~A}^{\prime}\right)$ & $7.55\left(\mathrm{~A}^{\prime}\right)$ & $7.25\left(\mathrm{~A}^{\prime}\right)$ & 7.33 & 7.33 \\
\hline
\end{tabular}


Table S14: Glycine CCSD ionisation (eV), aCDZ

\begin{tabular}{cccccc}
\hline & $\mathrm{I}$ & II & III & IV & V \\
\hline $\mathrm{I}_{1}$ & $10.01\left(\mathrm{~A}^{\prime}\right)$ & $9.98\left(\mathrm{~A}^{\prime}\right)$ & $9.69\left(\mathrm{~A}^{\prime}\right)$ & 9.85 & 9.89 \\
$\mathrm{I}_{2}$ & $10.90\left(\mathrm{~A}^{\prime}\right)$ & $11.35\left(\mathrm{~A}^{\prime \prime}\right)$ & $11.45\left(\mathrm{~A}^{\prime}\right)$ & 10.80 & 10.80 \\
$\mathrm{I}_{3}$ & $12.06\left(\mathrm{~A}^{\prime \prime}\right)$ & $11.37\left(\mathrm{~A}^{\prime}\right)$ & $11.92\left(\mathrm{~A}^{\prime \prime}\right)$ & 12.02 & 11.98 \\
$\mathrm{I}_{4}$ & $13.53\left(\mathrm{~A}^{\prime \prime}\right)$ & $13.42\left(\mathrm{~A}^{\prime}\right)$ & $13.69\left(\mathrm{~A}^{\prime \prime}\right)$ & 13.89 & 13.96 \\
$\mathrm{I}_{5}$ & $14.37\left(\mathrm{~A}^{\prime}\right)$ & $13.79\left(\mathrm{~A}^{\prime \prime}\right)$ & $14.34\left(\mathrm{~A}^{\prime}\right)$ & 14.28 & 14.21 \\
\hline
\end{tabular}

Table S15: Glycine CC3 ionisation (eV), aCDZ

\begin{tabular}{cccccc}
\hline & $\mathrm{I}$ & $\mathrm{II}$ & $\mathrm{III}$ & $\mathrm{IV}$ & $\mathrm{V}$ \\
\hline $\mathrm{I}_{1}$ & $9.97\left(\mathrm{~A}^{\prime}\right)$ & $9.94\left(\mathrm{~A}^{\prime}\right)$ & $9.65\left(\mathrm{~A}^{\prime}\right)$ & 9.82 & 9.85 \\
$\mathrm{I}_{2}$ & $10.88\left(\mathrm{~A}^{\prime}\right)$ & $11.29\left(\mathrm{~A}^{\prime}\right)$ & $11.38\left(\mathrm{~A}^{\prime}\right)$ & 10.79 & 10.77 \\
$\mathrm{I}_{3}$ & $12.01\left(\mathrm{~A}^{\prime \prime}\right)$ & $11.31\left(\mathrm{~A}^{\prime \prime}\right)$ & $11.88\left(\mathrm{~A}^{\prime \prime}\right)$ & 11.99 & 11.94 \\
$\mathrm{I}_{4}$ & $13.41\left(\mathrm{~A}^{\prime \prime}\right)$ & $13.29\left(\mathrm{~A}^{\prime}\right)$ & $13.56\left(\mathrm{~A}^{\prime \prime}\right)$ & 13.80 & 13.84 \\
$\mathrm{I}_{5}$ & $14.27\left(\mathrm{~A}^{\prime}\right)$ & $13.59\left(\mathrm{~A}^{\prime \prime}\right)$ & $14.23\left(\mathrm{~A}^{\prime}\right)$ & 14.12 & 14.12 \\
\hline
\end{tabular}

Table S16: Glycine CCSD core ionisation (eV), aCDZ

\begin{tabular}{cccccc}
\hline & I & II & III & IV & V \\
\hline C1 & 294.68 & 294.98 & 294.69 & 294.65 & 294.67 \\
C2 & 297.92 & 297.53 & 297.92 & 298.00 & 297.94 \\
N3 & 408.56 & 409.40 & 408.63 & 408.49 & 408.65 \\
O4 & 542.71 & 542.08 & 542.60 & 542.67 & 542.60 \\
O5 & 544.22 & 543.51 & 544.27 & 544.23 & 544.15 \\
\hline
\end{tabular}

Table S17: Glycine CC3 core ionisation (eV), aCDZ

\begin{tabular}{cccccc}
\hline & I & II & III & IV & V \\
\hline C1 & 293.37 & 293.65 & 293.39 & 293.35 & 293.36 \\
C2 & 296.57 & 296.18 & 296.56 & 296.67 & 296.61 \\
N3 & 406.48 & 407.26 & 406.53 & 406.38 & 406.51 \\
O4 & 538.91 & 538.27 & 538.78 & 538.88 & 538.82 \\
O5 & 540.83 & 539.98 & 540.86 & 540.86 & 540.78 \\
\hline
\end{tabular}




\title{
Conformers, CCSD core excitations, intensities and term
}

\author{
symbols
}

Table S18: Glycine CCSD core excitations C1 (eV), aCDZ

\begin{tabular}{ccccccccccc}
\hline & \multicolumn{2}{c}{$\mathrm{I}$} & \multicolumn{2}{c}{$\mathrm{II}$} & \multicolumn{2}{c}{$\mathrm{III}$} & \multicolumn{2}{c}{$\mathrm{IV}$} & \multicolumn{2}{c}{$\mathrm{V}$} \\
& $\Delta \mathrm{E}$ & $f \times 100$ & $\Delta \mathrm{E}$ & $f \times 100$ & $\Delta \mathrm{E}$ & $f \times 100$ & $\Delta \mathrm{E}$ & $f \times 100$ & $\Delta \mathrm{E}$ & $f \times 100$ \\
\hline $\mathrm{S}_{1}$ & 290.79 & $0.96\left(\mathrm{~A}^{\prime}\right)$ & 290.63 & $1.07\left(\mathrm{~A}^{\prime}\right)$ & 290.79 & $0.95\left(\mathrm{~A}^{\prime}\right)$ & 290.61 & 0.91 & 290.57 & 0.91 \\
$\mathrm{~S}_{2}$ & 291.03 & $0.58\left(\mathrm{~A}^{\prime \prime}\right)$ & 291.19 & $1.38\left(\mathrm{~A}^{\prime \prime}\right)$ & 291.00 & $0.57\left(\mathrm{~A}^{\prime \prime}\right)$ & 291.18 & 0.62 & 291.31 & 0.59 \\
$\mathrm{~S}_{3}$ & 291.96 & $0.34\left(\mathrm{~A}^{\prime}\right)$ & 291.89 & $0.66\left(\mathrm{~A}^{\prime}\right)$ & 291.92 & $0.61\left(\mathrm{~A}^{\prime}\right)$ & 291.89 & 0.82 & 291.74 & 1.46 \\
$\mathrm{~S}_{4}$ & 291.98 & $1.19\left(\mathrm{~A}^{\prime \prime}\right)$ & 292.37 & $0.30\left(\mathrm{~A}^{\prime \prime}\right)$ & 292.00 & $1.10\left(\mathrm{~A}^{\prime \prime}\right)$ & 292.08 & 0.86 & 292.10 & 0.45 \\
$\mathrm{~S}_{5}$ & 292.41 & $0.65\left(\mathrm{~A}^{\prime}\right)$ & 292.59 & $0.06\left(\mathrm{~A}^{\prime}\right)$ & 292.51 & $0.52\left(\mathrm{~A}^{\prime}\right)$ & 292.35 & 0.52 & 292.41 & 0.41 \\
\hline
\end{tabular}

Table S19: Glycine CCSD core excitations C2 (eV), aCDZ

\begin{tabular}{ccccccccccc}
\hline & \multicolumn{2}{c}{ I } & \multicolumn{2}{c}{ II } & \multicolumn{2}{c}{ III } & \multicolumn{2}{c}{ IV } & \multicolumn{2}{c}{ V } \\
& $\Delta \mathrm{E}$ & $f \times 100$ & $\Delta \mathrm{E}$ & $f \times 100$ & $\Delta \mathrm{E}$ & $f \times 100$ & $\Delta \mathrm{E}$ & $f \times 100$ & $\Delta \mathrm{E}$ & $f \times 100$ \\
\hline $\mathrm{S}_{1}$ & 290.53 & $7.20\left(\mathrm{~A}^{\prime \prime}\right)$ & 290.64 & $7.01\left(\mathrm{~A}^{\prime \prime}\right)$ & 290.53 & $7.19\left(\mathrm{~A}^{\prime \prime}\right)$ & 290.61 & 7.22 & 290.56 & 7.17 \\
$\mathrm{~S}_{2}$ & 294.35 & $0.10\left(\mathrm{~A}^{\prime}\right)$ & 294.15 & $0.12\left(\mathrm{~A}^{\prime}\right)$ & 294.26 & $0.21\left(\mathrm{~A}^{\prime}\right)$ & 294.51 & 0.12 & 294.46 & 0.19 \\
$\mathrm{~S}_{3}$ & 295.23 & $0.14\left(\mathrm{~A}^{\prime}\right)$ & 295.18 & $0.02\left(\mathrm{~A}^{\prime}\right)$ & 295.44 & $0.01\left(\mathrm{~A}^{\prime}\right)$ & 295.37 & 0.13 & 295.48 & 0.02 \\
$\mathrm{~S}_{4}$ & 295.63 & $0.03\left(\mathrm{~A}^{\prime \prime}\right)$ & 295.23 & $0.00\left(\mathrm{~A}^{\prime \prime}\right)$ & 295.63 & $0.03\left(\mathrm{~A}^{\prime \prime}\right)$ & 295.92 & 0.03 & 295.74 & 0.20 \\
$\mathrm{~S}_{5}$ & 295.84 & $0.02\left(\mathrm{~A}^{\prime}\right)$ & 295.34 & $0.13\left(\mathrm{~A}^{\prime}\right)$ & 295.69 & $0.17\left(\mathrm{~A}^{\prime}\right)$ & 295.96 & 0.04 & 295.91 & 0.01 \\
\hline
\end{tabular}

Table S20: Glycine CCSD core excitations N3 (eV), aCDZ

\begin{tabular}{ccccccccccc}
\hline & \multicolumn{2}{c}{$\mathrm{I}$} & \multicolumn{2}{c}{$\mathrm{II}$} & \multicolumn{2}{c}{$\mathrm{III}$} & \multicolumn{2}{c}{$\mathrm{IV}$} & \multicolumn{2}{c}{$\mathrm{V}$} \\
& $\Delta \mathrm{E}$ & $f \times 100$ & $\Delta \mathrm{E}$ & $f \times 100$ & $\Delta \mathrm{E}$ & $f \times 100$ & $\Delta \mathrm{E}$ & $f \times 100$ & $\Delta \mathrm{E}$ & $f \times 100$ \\
\hline $\mathrm{S}_{1}$ & 404.30 & $0.60\left(\mathrm{~A}^{\prime}\right)$ & 404.31 & $0.67\left(\mathrm{~A}^{\prime}\right)$ & 404.25 & $0.61\left(\mathrm{~A}^{\prime}\right)$ & 404.13 & 0.76 & 404.14 & 0.72 \\
$\mathrm{~S}_{2}$ & 405.41 & $1.89\left(\mathrm{~A}^{\prime \prime}\right)$ & 405.48 & $2.10\left(\mathrm{~A}^{\prime \prime}\right)$ & 405.38 & $1.97\left(\mathrm{~A}^{\prime \prime}\right)$ & 405.53 & 1.62 & 405.46 & 1.87 \\
$\mathrm{~S}_{3}$ & 405.94 & $0.10\left(\mathrm{~A}^{\prime}\right)$ & 406.50 & $0.14\left(\mathrm{~A}^{\prime}\right)$ & 406.04 & $0.07\left(\mathrm{~A}^{\prime}\right)$ & 405.93 & 0.27 & 406.08 & 0.17 \\
$\mathrm{~S}_{4}$ & 406.49 & $0.32\left(\mathrm{~A}^{\prime \prime}\right)$ & 406.70 & $0.75\left(\mathrm{~A}^{\prime}\right)$ & 406.53 & $0.24\left(\mathrm{~A}^{\prime \prime}\right)$ & 406.14 & 0.29 & 406.33 & 0.63 \\
$\mathrm{~S}_{5}$ & 406.57 & $0.59\left(\mathrm{~A}^{\prime}\right)$ & 407.49 & $0.44\left(\mathrm{~A}^{\prime}\right)$ & 406.55 & $0.37\left(\mathrm{~A}^{\prime}\right)$ & 406.68 & 0.86 & 406.64 & 1.24 \\
\hline
\end{tabular}


Table S21: Glycine CCSD core excitations O4 (eV), aCDZ

\begin{tabular}{ccccccccccc}
\hline & \multicolumn{2}{c}{ I } & \multicolumn{2}{c}{ II } & \multicolumn{2}{c}{ III } & \multicolumn{2}{c}{ IV } & \multicolumn{2}{c}{$\mathrm{V}$} \\
& $\Delta \mathrm{E}$ & $f \times 100$ & $\Delta \mathrm{E}$ & $f \times 100$ & $\Delta \mathrm{E}$ & $f \times 100$ & $\Delta \mathrm{E}$ & $f \times 100$ & $\Delta \mathrm{E}$ & $f \times 100$ \\
\hline $\mathrm{S}_{1}$ & 535.87 & $3.75\left(\mathrm{~A}^{\prime \prime}\right)$ & 535.80 & $3.63\left(\mathrm{~A}^{\prime \prime}\right)$ & 535.87 & $3.60\left(\mathrm{~A}^{\prime \prime}\right)$ & 535.89 & 3.71 & 535.80 & 3.74 \\
$\mathrm{~S}_{2}$ & 539.09 & $0.08\left(\mathrm{~A}^{\prime}\right)$ & 538.91 & $0.01\left(\mathrm{~A}^{\prime}\right)$ & 538.97 & $0.17\left(\mathrm{~A}^{\prime}\right)$ & 539.16 & 0.12 & 539.13 & 0.14 \\
$\mathrm{~S}_{3}$ & 540.01 & $0.44\left(\mathrm{~A}^{\prime}\right)$ & 539.74 & $0.16\left(\mathrm{~A}^{\prime}\right)$ & 540.11 & $0.33\left(\mathrm{~A}^{\prime}\right)$ & 540.06 & 0.42 & 540.15 & 0.33 \\
$\mathrm{~S}_{4}$ & 540.39 & $0.13\left(\mathrm{~A}^{\prime \prime}\right)$ & 539.85 & $0.12\left(\mathrm{~A}^{\prime \prime}\right)$ & 540.29 & $0.16\left(\mathrm{~A}^{\prime \prime}\right)$ & 540.59 & 0.12 & 540.49 & 0.17 \\
$\mathrm{~S}_{5}$ & 540.70 & $0.19\left(\mathrm{~A}^{\prime}\right)$ & 540.05 & $0.23\left(\mathrm{~A}^{\prime}\right)$ & 540.49 & $0.07\left(\mathrm{~A}^{\prime}\right)$ & 540.73 & 0.16 & 540.63 & 0.02 \\
\hline
\end{tabular}

Table S22: Glycine CCSD core excitations O5 (eV), aCDZ

\begin{tabular}{ccccccccccc}
\hline & \multicolumn{2}{c}{ I } & \multicolumn{2}{c}{ II } & \multicolumn{2}{c}{ III } & \multicolumn{2}{c}{ IV } & \multicolumn{2}{c}{$\mathrm{V}$} \\
& $\Delta \mathrm{E}$ & $f \times 100$ & $\Delta \mathrm{E}$ & $f \times 100$ & $\Delta \mathrm{E}$ & $f \times 100$ & $\Delta \mathrm{E}$ & $f \times 100$ & $\Delta \mathrm{E}$ & $f \times 100$ \\
\hline $\mathrm{S}_{1}$ & 539.19 & $1.56\left(\mathrm{~A}^{\prime}\right)$ & 539.17 & $1.90\left(\mathrm{~A}^{\prime \prime}\right)$ & 539.23 & $1.51\left(\mathrm{~A}^{\prime}\right)$ & 539.23 & 1.58 & 539.21 & 1.53 \\
$\mathrm{~S}_{2}$ & 539.75 & $1.46\left(\mathrm{~A}^{\prime \prime}\right)$ & 539.63 & $0.47\left(\mathrm{~A}^{\prime}\right)$ & 539.62 & $1.66\left(\mathrm{~A}^{\prime \prime}\right)$ & 539.73 & 1.49 & 539.68 & 1.50 \\
$\mathrm{~S}_{3}$ & 541.37 & $0.76\left(\mathrm{~A}^{\prime}\right)$ & 541.04 & $0.36\left(\mathrm{~A}^{\prime}\right)$ & 541.25 & $1.04\left(\mathrm{~A}^{\prime}\right)$ & 541.44 & 0.77 & 541.31 & 0.89 \\
$\mathrm{~S}_{4}$ & 541.74 & $0.37\left(\mathrm{~A}^{\prime \prime}\right)$ & 541.22 & $0.15\left(\mathrm{~A}^{\prime \prime}\right)$ & 541.80 & $0.28\left(\mathrm{~A}^{\prime \prime}\right)$ & 541.86 & 0.41 & 541.86 & 0.29 \\
$\mathrm{~S}_{5}$ & 542.05 & $0.36\left(\mathrm{~A}^{\prime}\right)$ & 541.31 & $0.27\left(\mathrm{~A}^{\prime}\right)$ & 542.03 & $0.38\left(\mathrm{~A}^{\prime}\right)$ & 542.21 & 0.30 & 542.20 & 0.41 \\
\hline
\end{tabular}




\section{Conformers, CC3 core excitations and term symbols}

Table S23: Glycine CC3 core excitations C1 (eV), aCDZ

\begin{tabular}{cccccc}
\hline & $\mathrm{I}$ & $\mathrm{II}$ & $\mathrm{III}$ & $\mathrm{IV}$ & $\mathrm{V}$ \\
\hline $\mathrm{S}_{1}$ & $289.77\left(\mathrm{~A}^{*}\right)$ & $289.57\left(\mathrm{~A}^{\prime}\right)$ & $289.74\left(\mathrm{~A}^{*}\right)$ & 289.53 & 289.46 \\
$\mathrm{~S}_{2}$ & $289.77\left(\mathrm{~A}^{*}\right)$ & $290.10\left(\mathrm{~A}^{\prime \prime}\right)$ & $289.76\left(\mathrm{~A}^{*}\right)$ & 289.99 & 290.12 \\
$\mathrm{~S}_{3}$ & $290.74\left(\mathrm{~A}^{\prime}\right)$ & $290.74\left(\mathrm{~A}^{\prime}\right)$ & $290.74\left(\mathrm{~A}^{\prime}\right)$ & 290.70 & 290.69 \\
$\mathrm{~S}_{4}$ & $290.83\left(\mathrm{~A}^{\prime \prime}\right)$ & $291.04\left(\mathrm{~A}^{\prime \prime}\right)$ & $290.85\left(\mathrm{~A}^{\prime \prime}\right)$ & 290.92 & 290.85 \\
$\mathrm{~S}_{5}$ & $291.06\left(\mathrm{~A}^{\prime}\right)$ & $291.37\left(\mathrm{~A}^{\prime}\right)$ & $291.14\left(\mathrm{~A}^{\prime}\right)$ & 291.06 & 291.07 \\
\hline
\end{tabular}

*Excitations are nearly degenerate and no symmetry can be determined.

Table S24: Glycine CC3 core excitations C2 (eV), aCDZ

\begin{tabular}{lccccc}
\hline & $\mathrm{I}$ & $\mathrm{II}$ & $\mathrm{III}$ & $\mathrm{IV}$ & $\mathrm{V}$ \\
\hline $\mathrm{S}_{1}$ & $290.15\left(\mathrm{~A}^{\prime \prime}\right)$ & $290.26\left(\mathrm{~A}^{\prime \prime}\right)$ & $290.16\left(\mathrm{~A}^{\prime \prime}\right)$ & 290.24 & 290.19 \\
$\mathrm{~S}_{2}$ & $293.09\left(\mathrm{~A}^{\prime}\right)$ & $292.78\left(\mathrm{~A}^{\prime}\right)$ & $292.98\left(\mathrm{~A}^{\prime}\right)$ & 293.25 & 293.19 \\
$\mathrm{~S}_{3}$ & $293.86\left(\mathrm{~A}^{\prime}\right)$ & $293.78\left(\mathrm{~A}^{\prime}\right)$ & $294.07\left(\mathrm{~A}^{\prime}\right)$ & 294.00 & 294.12 \\
$\mathrm{~S}_{4}$ & $294.35\left(\mathrm{~A}^{\prime \prime}\right)$ & $293.88\left(\mathrm{~A}^{\prime \prime}\right)$ & $294.30\left(\mathrm{~A}^{\prime}\right)$ & 294.60 & 294.38 \\
$\mathrm{~S}_{5}$ & $294.47\left(\mathrm{~A}^{\prime}\right)$ & $293.97\left(\mathrm{~A}^{\prime}\right)$ & $294.33\left(\mathrm{~A}^{\prime \prime}\right)$ & 294.65 & 294.61 \\
\hline
\end{tabular}

Table S25: Glycine CC3 core excitations N3 (eV), aCDZ

\begin{tabular}{cccccc}
\hline & $\mathrm{I}$ & $\mathrm{II}$ & $\mathrm{III}$ & $\mathrm{IV}$ & $\mathrm{V}$ \\
\hline $\mathrm{S}_{1}$ & $402.76\left(\mathrm{~A}^{\prime}\right)$ & $402.81\left(\mathrm{~A}^{\prime}\right)$ & $402.70\left(\mathrm{~A}^{\prime}\right)$ & 402.56 & 402.57 \\
$\mathrm{~S}_{2}$ & $403.76\left(\mathrm{~A}^{\prime \prime}\right)$ & $403.95\left(\mathrm{~A}^{\prime \prime}\right)$ & $403.81\left(\mathrm{~A}^{\prime \prime}\right)$ & 403.79 & 403.85 \\
$\mathrm{~S}_{3}$ & $403.94\left(\mathrm{~A}^{\prime}\right)$ & $404.56\left(\mathrm{~A}^{\prime}\right)$ & $403.94\left(\mathrm{~A}^{\prime \prime}\right)$ & 403.87 & 404.02 \\
$\mathrm{~S}_{4}$ & $403.94\left(\mathrm{~A}^{\prime \prime}\right)$ & $404.90\left(\mathrm{~A}^{\prime}\right)$ & $404.05\left(\mathrm{~A}^{\prime}\right)$ & 403.94 & 404.07 \\
$\mathrm{~S}_{5}$ & $404.55\left(\mathrm{~A}^{\prime}\right)$ & $405.10\left(\mathrm{~A}^{\prime \prime}\right)$ & $404.47\left(\mathrm{~A}^{\prime}\right)$ & 404.49 & 404.55 \\
\hline
\end{tabular}


Table S26: Glycine CC3 core excitations O4 (eV), aCDZ

\begin{tabular}{lccccc}
\hline & $\mathrm{I}$ & $\mathrm{II}$ & $\mathrm{III}$ & $\mathrm{IV}$ & $\mathrm{V}$ \\
\hline $\mathrm{S}_{1}$ & $533.94\left(\mathrm{~A}^{\prime \prime}\right)$ & $533.79\left(\mathrm{~A}^{\prime \prime}\right)$ & $533.84\left(\mathrm{~A}^{\prime \prime}\right)$ & 533.93 & 533.84 \\
$\mathrm{~S}_{2}$ & $535.63\left(\mathrm{~A}^{\prime}\right)$ & $535.12\left(\mathrm{~A}^{\prime}\right)$ & $535.44\left(\mathrm{~A}^{\prime}\right)$ & 535.69 & 535.64 \\
$\mathrm{~S}_{3}$ & $536.43\left(\mathrm{~A}^{\prime}\right)$ & $536.08\left(\mathrm{~A}^{\prime}\right)$ & $536.49\left(\mathrm{~A}^{\prime}\right)$ & 536.45 & 536.54 \\
$\mathrm{~S}_{4}$ & $536.83\left(\mathrm{~A}^{\prime \prime}\right)$ & $536.14\left(\mathrm{~A}^{\prime \prime}\right)$ & $536.71\left(\mathrm{~A}^{\prime \prime}\right)$ & 537.01 & 536.82 \\
$\mathrm{~S}_{5}$ & $537.01\left(\mathrm{~A}^{\prime}\right)$ & $536.29\left(\mathrm{~A}^{\prime}\right)$ & $536.75\left(\mathrm{~A}^{\prime}\right)$ & 537.02 & 537.00 \\
\hline
\end{tabular}

Table S27: Glycine CC3 core excitations O5 (eV), aCDZ

\begin{tabular}{lccccc}
\hline & $\mathrm{I}$ & $\mathrm{II}$ & $\mathrm{III}$ & $\mathrm{IV}$ & $\mathrm{V}$ \\
\hline $\mathrm{S}_{1}$ & $536.86\left(\mathrm{~A}^{\prime \prime}\right)$ & $536.44\left(\mathrm{~A}^{\prime \prime}\right)$ & $536.84\left(\mathrm{~A}^{\prime \prime}\right)$ & 536.86 & 536.78 \\
$\mathrm{~S}_{2}$ & $536.92\left(\mathrm{~A}^{\prime}\right)$ & $536.65\left(\mathrm{~A}^{\prime}\right)$ & $536.92\left(\mathrm{~A}^{\prime}\right)$ & 536.99 & 536.97 \\
$\mathrm{~S}_{3}$ & $538.30\left(\mathrm{~A}^{\prime}\right)$ & $537.72\left(\mathrm{~A}^{\prime}\right)$ & $538.35\left(\mathrm{~A}^{\prime}\right)$ & 538.41 & 538.38 \\
$\mathrm{~S}_{4}$ & $538.74\left(\mathrm{~A}^{\prime \prime}\right)$ & $537.83\left(\mathrm{~A}^{\prime \prime}\right)$ & $538.75\left(\mathrm{~A}^{\prime \prime}\right)$ & 538.91 & 538.76 \\
$\mathrm{~S}_{5}$ & $538.90\left(\mathrm{~A}^{\prime}\right)$ & $537.85\left(\mathrm{~A}^{\prime}\right)$ & $538.82\left(\mathrm{~A}^{\prime}\right)$ & 539.01 & 538.99 \\
\hline
\end{tabular}




\section{References}

(1) Plekan, O.; Feyer, V.; Richter, R.; Coreno, M; de Simone, M; Prince, K. C.; Carravetta, V. Investigation of the Amino Acids Glycine, Proline, and Methionine by Photoemission Spectroscopy, J. Phys. Chem A, 111, 10998-11005, 2007

(2) Plekan, O.; Feyer, V.; Richter, R.; Coreno, M; de Simone, M; Prince, K. C.; Carravetta, V. An X-ray absorption study of glycine, methionine and proline, J. Electron Spectrosc., 155, 1-3, 2007 